\title{
The Elementary Gravitational Charge and Its Value
}

\author{
Tomas Kala \\ Faculty of Informatics and Management, University of Hradec Kralove, 500 03, Czech Republic
}

\begin{abstract}
In the article "The Gravitational Force Quantum and its Value” [1], the author defined a gravitational force of the atomic unit (“the Gravitational Force Quantum”) as a gravitational force which exerts one atomic unit of the Earth’s mass on 1 kilogram of a mass on the Earth's surface, and he calculated its value as: $\mathrm{GFQ}_{\text {Earth }}=1.4958 \times 10^{-54} \mathrm{~N}$. In the present contribution, he extended the Gravitational Force Quantum concept to further Objects of the Solar Planetary System and for the Pluto. He calculated values of the $\mathrm{GFQ}_{0}$ on the analogous basis, i.e. of the mass and the standard acceleration of the gravity of individual objects and of the atomic unit of the mass. He received GFQ values for the Mercury $102.1427 \times 10^{-55} \mathrm{~N}$, the Venus $16,60012 \times 10^{-55} \mathrm{~N}$, the Earth $14.97839 \times 10^{-55}$ $\mathrm{N}$, the Mars $52.91869 \times 10^{-55} \mathrm{~N}$, the Jupiter $0.124391 \times 10^{-55} \mathrm{~N}$, the Saturn $0.17929 \times 10^{-55} \mathrm{~N}$, the Uranus $0.945178 \times 10^{-55} \mathrm{~N}$, the Neptune $1.002845 \times 10^{-55} \mathrm{~N}$, for the Pluto $458.9124 \times 10^{-55} \mathrm{~N}$, and for the Sun $0.001257 \times 10^{-55} \mathrm{~N}$, respectively. He multiplied the GFQo values by second power of the radii of the individual objects $(\mathrm{O})$, receiving values denoted as the "Elementary Gravitational Charge" $\left(G_{O}\right)$. The Elementary Gravitational Charge represents a gravitational force of one atomic unit of mass in the (radius) distance of 1 meter. They were found of the same value: $G_{M e}=G_{V}=G_{E}=G_{M a}=G_{J}=G_{S}=G_{P}=G_{S u n}=6.079675463 \times 10^{-41} N$. The values were the same as the calculated one on the basis of the "classical” Newton's formula: $\mathrm{F}_{\mathrm{G}}=\boldsymbol{u} \times \mathrm{M} \times \mathrm{m} / \mathrm{R}^{2}$, for the gravitational force between the atomic unit mass and a mass of $1 \mathrm{~kg}$ at a distance of 1 meter, which value was calculated as $\mathrm{G}=6.079675463 \times$ $10^{-41} \mathrm{~N}$. The quantity of the Elementary Gravitational Charge can be supposed to be analogous to the Elementary (Electric) Charge (e $=1.6021766208(98) \times 10^{-19}$ C) quantity.
\end{abstract}

Key words: Gravitation, gravitational force of atomic unit, gravitational force quantum, elementary gravitational charge.

\section{Nomenclature}

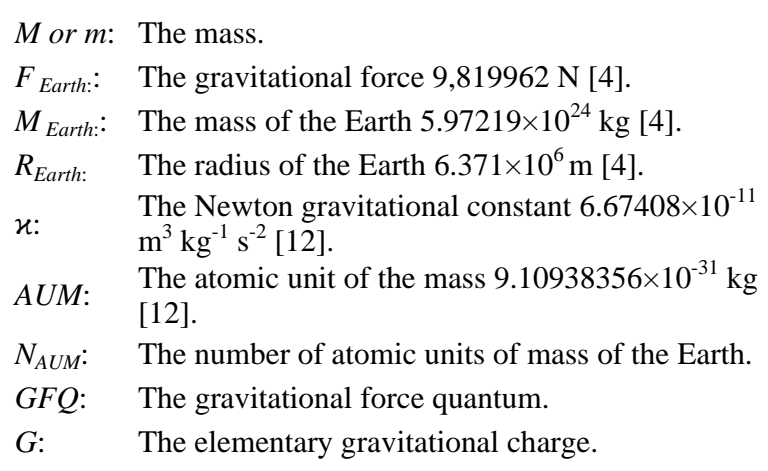

\section{Introduction}

In the previous article titled The Gravitational Force Quantum and its Value” [1], the author defined the gravitational force of the atomic unit (or “Gravitational Force Quantum”) as a gravitational force which exerts one atomic unit of the Earth's mass on $1 \mathrm{~kg}$ of a mass on the Earth's surface, and he

Corresponding author: Tomas Kala, Dr.Sc., assistent professor, research fields: material sciences and social sciences. calculated its value as: GFQ $=1.4958 \times 10^{-54} \mathrm{~N}$, respecting the obvious fact, that the gravitation/gravity is an additive property of matter objects and taking into account other positivistic quantities like the mass of the Earth, the standard acceleration of the gravity, and the value of the atomic unit of the mass.

In the present contribution, the author extended the Gravitational Force Quantum (GFQ) concept to further objects of the Sun planetary system and for Pluto.

The author recalculated the values found by multiplying them by second power of the radii of the corresponding objects, receiving values, which he defined as the "Elementary Gravitational Charge" $\left(\mathrm{G}_{\mathrm{O}}\right)$ of the (one) atomic unit of mass of individual objects in the (radius) distance of 1 meter. He also calculated the Elementary Gravitational Charge $(G)$ on the basis of the classical Newton's law for the atomic unit of the mass. 


\section{Methodology}

According to the Newton's law, a mass of $\mathrm{m}=1 \mathrm{~kg}$ exerts on the Earth's surface a gravitational force $\mathrm{F}_{\text {Earth }}$ $=9.819962 \mathrm{~N}$.

The mass of the Earth $\left(5.97219 \times 10^{24} \mathrm{~kg}[4]\right)$ can be expressed as:

$$
\mathrm{M}_{\text {Earth }}=\mathrm{AUM} \times \mathrm{N}_{\mathrm{AUMEarth}}
$$

where the AUM is the atomic unit of the mass, $9.10938356 \times 10^{-31} \mathrm{~kg}$ [12]), the $\mathrm{N}_{\text {AUMEarth }}$ is a number of atomic units of the mass contained in the Earth, i.e.:

$$
\begin{gathered}
\mathrm{N}_{\text {AUMEarth }}=\mathrm{M}_{\text {Earth }} / \mathrm{AUM}= \\
5.97219 \times 10^{24} / 9.10938356 \times 10^{-31}=0.655609 \times 10^{55}
\end{gathered}
$$

The value of the gravitational force attributed to one atomic unit of the Earth's mass, i.e. a "GFQEarth (the Gravitational force quantum)” can be calculated as:

$$
\begin{aligned}
& \mathrm{GFQ}_{\text {Earth }}=\mathrm{F}_{\text {Earth }} / \mathrm{N}_{\mathrm{AUM}}= \\
& 9.819962 / 0.655609 \times 10^{55}= \\
& 1.497839 \times 10^{-54} \mathrm{~N}
\end{aligned}
$$

The value of the "Elementary Gravitational Charge" $\left(\mathrm{G}_{\mathrm{O}}\right)$ of the Earth was calculated as:

$$
\begin{gathered}
\mathrm{G}_{\text {Earth }}=\mathrm{GFQ}_{\text {Earth }} \times \mathrm{R}_{\text {Earth }}{ }^{2}= \\
1.497839 \times 10^{-54} \times\left(6.371 \times 10^{6}\right)^{2}= \\
6.079675463 \times 10^{-41} \mathrm{~N}
\end{gathered}
$$

The same procedures were followed with data for the planets of the solar system and Pluto given in the Table 1.

The value of the "Elementary Gravitational Charge" corresponding to the atomic unit of the mass
$\mathrm{G}_{\text {AUM }}$ was calculated by definition as a gravitational force between the atomic unit mass and a mass of $\mathrm{m}=1 \mathrm{~kg}$ at a distance of 1 meter using the formula:

$$
\begin{gathered}
\mathrm{G}_{\mathrm{AUM}}=\mathcal{x} \times \mathrm{M}_{\mathrm{AUM}} \times \mathrm{m} / \mathrm{R}^{2} \\
=6.67408 \times 10^{-11} \times 9.10938356 \times 10^{-31} \times 1 / 1^{2} \\
=6.079675463 \times 10^{-41} \mathrm{~N} .
\end{gathered}
$$

\section{Results and Discussion}

The author calculated values of the GFQo on the analogous basis, i.e. of the mass and the standard acceleration of the gravity of individual objects and of the atomic unit of the mass given in the Table 1.

He received GFQo values for: the Mercury $102.1427 \times 10^{-55} \mathrm{~N}$, the Venus $16.60012 \times 10^{-55} \mathrm{~N}$, the Earth $14.97839 \times 10^{-55} \mathrm{~N}$, the Mars $52.91869 \times 10^{-55}$ $\mathrm{N}$, the Jupiter $0.124391 \times 10^{-55} \mathrm{~N}$, the Saturn 0.17929 $\times 10^{-55} \mathrm{~N}$, the Uranus $0.945178 \times 10^{-55} \mathrm{~N}$, the Neptune $1.002845 \times 10^{-55} \mathrm{~N}$, for the Pluto $458.9124 \times 10^{-55} \mathrm{~N}$, and for the Sun $0.001257 \times 10^{-55} \mathrm{~N}$, respectively (see the Table 1).

The GFQo values were multiplied by the square roots of the radii of the corresponding objects, receiving values, which were defined as the "Elementary Gravitational Charge” $\left(\mathrm{G}_{\mathrm{O}}\right)$ of the (one) atomic unit of each object's mass in the radius distance of 1 meter”. They were found (see the Table 1) of the same values, i.e. $\mathrm{G}_{\mathrm{Me}}=\mathrm{G}_{\mathrm{V}}=\mathrm{G}_{\mathrm{E}}=\mathrm{G}_{\mathrm{Ma}}=\mathrm{G}_{\mathrm{J}}=\mathrm{G}_{\mathrm{S}}=\mathrm{G}_{\mathrm{P}}$ $=\mathrm{G}_{\text {Sun }}=6.079675463 \times 10^{-41} \mathrm{~N}$.

Table 1 Values of Mass $\left(\mathbf{M}_{\mathrm{O}}\right)$, Radius $\left(\mathrm{R}_{\mathrm{O}}\right)$, Gravity $\left(\mathrm{F}_{\mathrm{O}}\right)$, Number of atomic units $\left(\mathrm{N}_{\mathrm{AUMO}}\right)$, Gravitational Force Quantum (GFQo), and Elementary Gravitational Charge $\left(G_{0}\right)$ of individual Objects of the Solar Planetary System and for Pluto.

\begin{tabular}{lllllll}
\hline Object & $\mathrm{M}_{\mathrm{O}} \times 10^{24} \mathrm{~kg}$ & $\mathrm{R}_{\mathrm{O}}, \mathrm{km}$ & $\mathrm{F}_{\mathrm{O}}, \mathrm{ms}^{-2}$ & $\mathrm{~N}_{\mathrm{AUMO}} \times 10^{55}$ & $\mathrm{GFQ}_{\mathrm{O}} \times 10^{-55} \mathrm{~N}$ & $\mathrm{G}_{\mathrm{O}} \times 10^{-41} \mathrm{~N}$ \\
\hline Mercury & 0.330104 & 2439.7 & 3.701428 & 0.036238 & 102.1427 & 6.079675463 \\
Venus & 4.86732 & 6051.8 & 8.869766 & 0.534319 & 16.60012 & 6.079675463 \\
Earth & 5.97219 & 6371 & 9.819962 & 0.655609 & 14.97839 & 6.079675463 \\
Mars & 0.641693 & 3389.5 & 3.727756 & 0.070443 & 52.91869 & 6.079675463 \\
Jupiter & 1898.13 & 69911 & 25.91948 & 208.3709 & 0.124391 & 6.079675463 \\
Saturn & 568.319 & 58232 & 11.18562 & 62.38831 & 0.17929 & 6.079675463 \\
Uranus & 86.8103 & 25362 & 9.007322 & 9.529767 & 0.945178 & 6.079675463 \\
Neptune & 102.41 & 24622 & 11.27424 & 11.24225 & 1.002845 & 6.079675463 \\
Pluto & 0.1309 & 1151 & 6.594478 & 0.01437 & 458.9124 & 6.079675463 \\
Sun & 1989100 & 695508 & 274.4377 & 218357.3 & 0.001257 & 6.079675463 \\
Sources & {$[2-11]$} & {$[2-11]$} & Own calcul. & Own calcul. & Own calcul. & Own calcul. \\
\hline
\end{tabular}


These values were the same as the one, which was calculated on the basis of the "classical" Newton's formula: $F_{G}=\varkappa \times M \times m / R^{2}$, for the gravitational force between the atomic unit mass and a mass of $1 \mathrm{~kg}$ at a distance of 1 meter, i.e. $6.079675463 \times 10^{-41} \mathrm{~N}$.

The verity of the values of the GFQo and $G_{0}$ might be limited by correctness of the atomic unit of the mass, the objects' gravitational forces, mass and radius values, which were used in the calculation of the gravitational force quantum and elementary gravitational charge values. It can be foreseen, that the value of GFQ and $G_{A U M}$ will be revised in the future development of notion.

\section{Conclusions}

The gravitation/gravity should be considered as one of the immanent/in-born/distinguished property of the matter, like:

- a mass or a weight,

- an inertia,

- a space/volume,

- a structure,

- electric charge,

- and others, which were discussed in the work [1].

Respecting the obvious fact, that the gravitation/gravity is an additive property of matter objects and taking into account other positivistic quantities like the mass, and the radius of the Earth, and other objects of the Sun planetary system and the Pluto, and the value of the atomic unit of the mass, the author defined the gravitational force of the atomic unit (or “Gravitational Force Quantum”) as a gravitational force which exerts one atomic unit of the objects' mass on $1 \mathrm{~kg}$ of a mass on the corresponding objects' surface, and he calculated their values as for the Mercury $102.1427 \times 10^{-55} \mathrm{~N}$, the Venus $16,60012 \times 10^{-55} \mathrm{~N}$, the Earth $14.97839 \times 10^{-55} \mathrm{~N}$, the Mars $52.91869 \times 10^{-55} \mathrm{~N}$, the Jupiter $0.124391 \times 10^{-55} \mathrm{~N}$, the Saturn $0.17929 \times$ $10^{-55} \mathrm{~N}$, the Uranus $0.945178 \times 10^{-55} \mathrm{~N}$, the Neptune $1.002845 \times 10^{-55} \mathrm{~N}$, for the Pluto $458.9124 \times 10^{-55} \mathrm{~N}$, and the Sun $0.001257 \times 10^{-55} \mathrm{~N}$, respectively.
The author defined the Elementary Gravitational Charge $(G)$ as a force which exerts one atomic unit of the mass on $1 \mathrm{~kg}$ of the mass in the distance of 1 meter, which value was calculated as $6.079675463 \times 10^{-41} \mathrm{~N}$.

The parameter of the Elementary Gravitational Charge can be supposed to be analogous to the Elementary (Electric) Charge e $=1.6021766208$ (98) $\mathrm{x} 10^{-19} \mathrm{C}$ [13]. Namely, both of the quantities cannot be divided into smaller parts, both are additive, both can be measured on the basis of the force effects, both are well known from their apparent physical features and practical applications, however, origins and physical backgrounds of the both phenomena are hidden so far [13-15].

The quantity of the Elementary Gravitational Charge will probably be useful for the further development of the "quantum mechanical” approach to the description and to the general notion about the world.

The phenomenon of the gravitation might perhaps be connected with/caused/explained/etc. by the distortion of the space around the mass objects. It could be one of the main in-born/decisive/distinguish characteristics of material objects (or a matter/mass). For further (rather philosophical) discussion, see ref. [15].

\section{References}

[1] Kala, T. 2015. "The Gravitational Force Quantum and its Value.” Journal of Physical Science and Application 5 (4): 288-90.

[2] NASA. "Earth: by the Numbers.” Accessed Feb. 5, 2016. https://web.archive.org/web/20150215052645/http://solar system.nasa.gov/planets/profile.cfm?Object=Mercury\&D isplay=Facts.

[3] NASA. "Earth: by the Numbers.” Accessed Feb. 5, 2016. https://web.archive.org/web/20150217022731/http://solar system.nasa.gov/planets/profile.cfm?Object=Venus\&Dis play=Facts.

[4] NASA. "Earth: by the Numbers.” Accessed Feb. 5, 2016. https://web.archive.org/web/20150217142912/http://solar system.nasa.gov/planets/profile.cfm?Object=Earth\&Displ ay=Facts.

[5] NASA. "Earth: by the Numbers." Accessed Feb. 5, 2016. https://web.archive.org/web/20150217075323/http://solar system.nasa.gov/planets/profile.cfm?Object=Mars\&Displ ay=Facts. 
[6] NASA. "Earth: by the Numbers.” Accessed Feb. 5, 2016. https://web.archive.org/web/20150215072818/http://solar system.nasa.gov/planets/profile.cfm?Object=Jupiter\&Dis play=Facts.

[7] NASA. "Earth: by the Numbers.” Accessed Feb. 5, 2016. https://web.archive.org/web/20150215075328/http://solar system.nasa.gov/planets/profile.cfm?Object=Saturn\&Dis play=Facts.

[8] NASA. "Earth: by the Numbers.” Accessed Feb. 5, 2016. https://web.archive.org/web/20150219021122/http://solar system.nasa.gov/planets/profile.cfm?Object=Uranus\&Dis play=Facts.

[9] NASA. "Earth: by the Numbers.” Accessed Feb. 5, 2016. https://web.archive.org/web/20150224013840/http://solar system.nasa.gov/planets/profile.cfm?Object=Neptune\&Di splay=Facts.

[10] NASA. "Earth: by the Numbers.” Accessed Feb. 5, 2016. https://web.archive.org/web/20150206201510/http://solar system.nasa.gov/planets/profile.cfm?Object=Pluto

[11] NASA. "Earth: by the Numbers.” Accessed Feb. 5, 2016. https://web.archive.org/web/20150207205115/ http://solarsystem.nasa.gov/planets/profile.cfm?Object=S un.

[12] NIST. "Fundamental Physical Constants - Complete Listing.” Accessed Feb 5, 2016. http://physics.nist.gov/cuu/Constants/Table/allascii.txt.

[13] Wikipedia. "Electric Charge." Accessed Feb. 5, 2016. https://en.wikipedia.org/wiki/Electric_charge.

[14] Halliday, D. and Resnick, R. 1981. "Fundamentals of Physics.” 2nd Edition, John Wiley \& Sons, Inc. N.Y., Chichester, Brisbane, Toronto.

[15] Kala, T. 2015 "Comments on Matter/Mass/Field Categories.” Unitary Theory of the World. Accessed Feb. 5, 2016. http://www.tomas-kala.net/. 